\title{
STUDY OF LHCb PENTAQUARKS WITH CLAS12*
}

\author{
Andrea Celentano \\ INFN Genova - Istituto Nazionale di Fisica Nucleare \\ Via Dodecaneso, 33, 16146 Genova, Italy
}

(Received October 2, 2017)

\begin{abstract}
The experimental study of the $J / \psi$ photoproduction near threshold is an attractive subject for studying the nucleon gluonic form-factor and, as has been shown recently, is a direct way to produce and study hiddencharm pentaquark states claimed by the LHCb, $P_{c}(4380)$ and $P_{c}(4450)$. The CLAS12 experiment in Hall B at Jefferson Laboratory has developed a program to measure near-threshold $J / \psi$ photoproduction using the low- $Q^{2}$ electroproduction technique. Given the foreseen resolution in the invariant mass of the $p-J / \psi$ system and the expected production rate for the pentaquark states, the experiment will be able to unambiguously confirm the existence of these states, measure the corresponding line-shape, and determine their quantum numbers.
\end{abstract}

DOI:10.5506/APhysPolBSupp.10.1043

\section{Introduction}

Since its discovery, the $J / \psi$ meson has been a subject of intense experimental studies, in particular at $e^{+} e^{-}$colliders. The photo- and electroproduction of $J / \psi$ on the nucleon has been studied mostly at large energies, where the production occurs mainly in the forward direction, at low values of transferred momentum. The only few data points for the production cross section close to threshold were obtained from experiments performed at SLAC [1] and Cornell [2], and show some discrepancies with each other.

Other than giving access to the local color (gluon) fields in the nucleon, thus revealing properties such as their response to momentum transfer, their spatial distribution, and their correlation with valence quark, this kinematic region is of particular interest given the recent claim by the LHCb Collaboration [3] of two hidden-charm pentaquark states, the so-called $P_{c}(4380)$ and $P_{c}(4450)$ states. If such states exist, and thus the origin of the LHCb signal are two genuine resonances in the $p-J / \psi$ system, they should manifest as $s$-channel poles in the reaction $\gamma p \rightarrow p J / \psi$.

* Presented at "Excited QCD 2017", Sintra, Lisbon, Portugal, May 7-13, 2017. 
Thanks to the $11-\mathrm{GeV} e^{-}$beam from the CEBAF accelerator, the CLAS12 experiment in Hall B at Jefferson Laboratory can efficiently explore this hitherto unknown region. The CLAS12 physics program includes a dedicated effort to study $J / \psi$ photoproduction near threshold, the E12-12-001A experiment [4]. This will study $J / \psi$ photoproduction in the energy region from the production threshold, $8.2 \mathrm{GeV}$, to the maximum available beam energy using two complementary techniques: "untagged photoproduction" and "tagged quasi-real photoproduction", i.e. low- $Q^{2}$ electroproduction.

\section{Physics motivation}

The LHCb Collaboration claimed the observation of two pentaquark states in the $\Lambda_{b} \rightarrow p J / \psi K^{-}$decay channel. One state has a mass of $4380 \pm 8 \pm 29 \mathrm{MeV}$ and a width of $205 \pm 18 \pm 86 \mathrm{MeV}$, while the second is narrower, with a mass of $4449 \pm 1.7 \pm 2.5 \mathrm{MeV}$ and a width of $39 \pm 5 \pm 19 \mathrm{MeV}$. The preferred $J^{P}$ assignments are of opposite parity, with one state having spin $3 / 2$ and the other $5 / 2$. However, the original paper does not reject other quantum number assignments by exchanging parity or spin of the two states. The narrow $P_{c}(4450)$ state is visible as a peak in the $p-J / \psi$ invariant mass spectrum, while the evidence for the other state $P_{c}(4380)$ was found after rather complicated data analysis.

Immediately after the LHCb claim, many authors proposed models to explain the microscopic structure of these states. Discussed options include composite charmonium-nucleon states [5], hybrid molecules [6], tightly correlated diquarks [7] or colored baryon-like and meson-like constituents [8]. Finally, it has also been suggested that at least one of the peaks is not a resonance at all, but rather a kinematical singularity due to rescattering effects [9]. Clearly, resolving between the models and clarifying the nature of the discovered hidden-charm pentaquark peaks requires further experimental studies. Since these states were observed in the decay mode $p-J / \psi$, it is natural to expect that they can be produced in the photoproduction process $\gamma p \rightarrow p J / \psi$, where they would appear as $s$-channel resonances at photon energy around $10 \mathrm{GeV}$ (see Fig. 1).

This production mode can be advantageous for detailed studies of the production and decay properties of the pentaquark resonances in comparison with the $\mathrm{LHCb}$ environment. The yield is determined by the branching fraction $\operatorname{Br}\left(P_{c} \rightarrow \gamma p\right) \equiv \mathrm{Br}_{\text {in }}$, and can be expressed in terms of $\operatorname{Br}\left(P_{c} \rightarrow\right.$ $p J / \psi) \equiv \mathrm{Br}_{\text {out }}$ by a relation similar to vector dominance for the $J / \psi$ photoproduction, as was shown in [5]. Although such dominance cannot be justified as a general rule, in the situation at hand, it can be applied due to arguments based on the heavy quark properties and special kinematics of the involved processes. As a result, the peak cross section for the $P_{c}$ photo- 

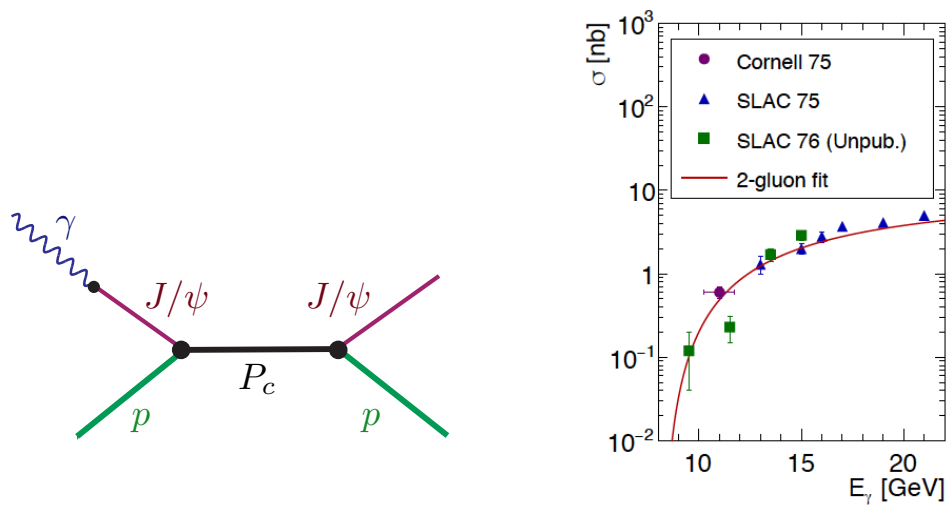

Fig. 1. Left: tree-level Feynmann diagram for the pentaquark photoproduction process. Right: existing data for $J / \psi$ photoproduction on the nucleon near threshold. The solid curve is a fit with the model from Ref. [10].

production process is proportional to $\mathrm{Br}_{\text {out }}^{2}$ and can reach tens of nanobarns or more, if $\operatorname{Br}\left(P_{c} \rightarrow p J / \psi\right) \simeq 1 \div 10 \%$. Such a relatively large cross section allows fairly detailed studies of the pentaquarks and a search for other similar states, for relatively moderate integrated luminosity.

\subsection{Pentaquark photoproduction cross section}

For a resonance $P_{c}$ in the $s$-channel, the photoproduction cross section of the reaction $\sigma\left(\gamma+p \rightarrow P_{c} \rightarrow J / \psi+p\right)$ is given by the standard Breit-Wigner expression (see e.g. in Ref. [11], Sec. 48.1.)

$$
\sigma(W)=\frac{2 J+1}{4} \frac{4 \pi}{k^{2}} \frac{\Gamma^{2} / 4}{\left(W-M_{c}\right)^{2}+\Gamma^{2} / 4} \operatorname{Br}_{\mathrm{in}} \mathrm{Br}_{\mathrm{out}}
$$

where $J$ is the spin of the $P_{c}$ resonance, $W=\sqrt{s}$ is the center-of-mass (c.m.) energy, $M_{c}$ and $\Gamma$ are the resonance mass and width respectively, and $k$ is the c.m. momentum of the colliding particles.

The actual value of the cross section in Eq. (1), naturally, depends on the product of the branching fractions, neither of which is presently known. However, the branching fraction $\operatorname{Br}\left(P_{c} \rightarrow \gamma+p\right)$ can be estimated in terms of $\operatorname{Br}\left(P_{c} \rightarrow J / \psi+p\right)$ in a way that does not directly rely on a specific model of the internal dynamics of the pentaquarks, but which is somewhat sensitive to the structure of the amplitude of the decay $P_{c} \rightarrow J / \psi+p$.

It was shown in Ref. [5] that the bounds for the formation cross section at the resonance maximum assuming the quantum numbers $J^{P}=(3 / 2)^{-}$ and $(5 / 2)^{+}$, respectively, for $P_{c}(4380)$ and $P_{c}(4450)$ are 


$$
\begin{aligned}
& P_{c}(4380): 1.5 \mu \text { barn }<\sigma_{0} /\left(\mathrm{Br}_{\text {out }}^{2}\right)<50 \mu \text { barn } \\
& P_{c}(4450): 12 \mu \text { barn }<\sigma_{0} /\left(\mathrm{Br}_{\text {out }}^{2}\right)<360 \mu \text { barn }
\end{aligned}
$$

where the lower bound corresponds to the presence of only the lower allowed partial wave, while the upper bound is found in the opposite situation where only the higher orbital wave is present. Currently, neither branching fraction $\operatorname{Br}\left(P_{c} \rightarrow J / \psi+p\right)$ is known nor the spin-parity quantum numbers for the observed pentaquarks are determined with certainty. Thus, it is not possible to estimate more precisely the value of the discussed formation cross section in a model-independent way.

\subsection{Electroproduction cross section}

The cross section for exclusive $J / \psi$ electroproduction can be decomposed in the sum of transversely $\left(\sigma_{\mathrm{T}}\right)$, and longitudinally $\left(\sigma_{\mathrm{L}}\right)$ polarized photon cross sections

$$
\frac{\mathrm{d} \sigma_{e p \rightarrow e^{\prime} J / \psi p^{\prime}}}{\mathrm{d} Q^{2} \mathrm{~d} W \mathrm{~d} t}=\Gamma_{\mathrm{T}}\left(\frac{\mathrm{d} \sigma_{\mathrm{T}}}{\mathrm{d} t}+\varepsilon \frac{\mathrm{d} \sigma_{\mathrm{L}}}{\mathrm{d} t}\right) .
$$

Here, $\Gamma_{\mathrm{T}}$ represents the flux of transverse virtual photons, while $\varepsilon$ is the ratio of longitudinal $\Gamma_{\mathrm{L}}$ to transverse $\Gamma_{\mathrm{T}}$ virtual photon fluxes. Using vector meson dominance (VDM), one can relate $\sigma_{\mathrm{T}}$ and $\sigma_{\mathrm{L}}$ to the photoproduction cross section $\sigma_{\gamma}$ [12]. The relation is simplified from the fact that, in the proposed measurement, $Q^{2}<0.2 \mathrm{GeV}^{2}$, and therefore one has $\sigma_{\mathrm{L}} \approx 0$ and $\sigma_{\mathrm{T}} \approx \sigma_{\gamma}$. This equation thus permits to relate the low- $Q^{2}$ electroproduction measurement with the corresponding photoproduction one.

\section{CLAS12 pentaquark searches}

In the aforementioned CLAS12 experiments, the reaction $e p \rightarrow e^{\prime} p J / \psi$ will be measured by detecting different final states, affected by different backgrounds, identifying the $J / \psi$ meson and the charmed pentaquarks using the missing mass or the invariant mass analysis.

In the tagged photoproduction case, the scattered electron will be detected in the CLAS12 Forward Tagger (FT), while recoil proton and leptons from the $J / \psi$ decay ( $e$ or $\mu$ ) will be detected in the CLAS12 Forward Detector (FD). In this case, the $J / \psi$ will be identified using the missing mass technique by measuring the scattered electron and recoil proton, while the pentaquarks will be identified in the $W$ distribution calculated from the scattered electron momentum. In the untagged electroproduction case, instead, the recoil proton and both decay leptons will be detected in FD. The $J / \psi$ will be reconstructed by measuring the lepton pair invariant mass, while pentaquarks will be identified in the invariant mass of $p-J / \psi$ system. 
The effective luminosity of the two approaches is shown in Fig. 2 for the nominal CLAS12 $e^{-}$luminosity of $10^{35} \mathrm{~cm}^{-2} \mathrm{~s}^{-1}$. In the energy range from $J / \psi$ production threshold to $11 \mathrm{GeV}$, the untagged photon flux is an order of magnitude larger than the tagged photon flux.
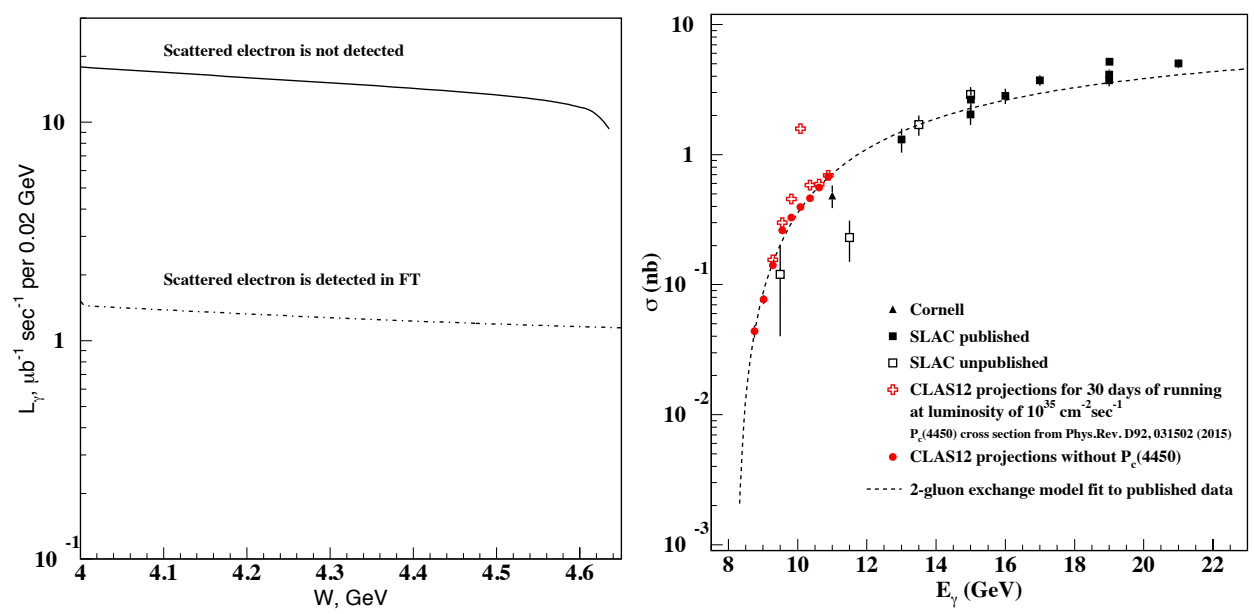

Fig. 2. (Color online) Left: comparison of effective luminosities for untagged and tagged electroproduction cases. Right: the total cross section as a function of photon energy for $J / \psi$ photoproduction. The gray/red points are expected CLAS12 results with (open crosses) and without (filled circles) $P_{c}(4450)$ pentaquark, summing yields from the tagged and the untagged measurements. The cross section based on the two gluon exchange mechanism was used to estimate rates, dashed line. The black points are existing data in this energy region from SLAC and Cornell.

Having a good invariant mass resolution for the $p-J / \psi$ system is crucial in order to identify a possible resonance peak. In the untagged case, the $W$ resolution is about $14 \mathrm{MeV}$ at the resonance peak for the full field setting of the CLAS12 torus magnet and increases for lower field values. In the tagged case, instead, $W$ is determined by the missing mass of the final-state electron that depends only on the measured electron energy in the Forward Tagger detector. This resolution (independent of the torus field setting) is about $5 \mathrm{MeV}$ at the $P_{c}(4450)$ resonance peak. Both values are smaller than the width of the narrower $P_{c}$ state claimed by the LHCb, thus allowing a proper determination of the line-shape of these resonances. 
The foreseen yield of detected pentaquark events per day, at the nominal CLAS12 luminosity, is reported in Table I, assuming $\operatorname{Br}\left(P_{c} \rightarrow p J / \psi\right)=1 \%$.

TABLE I

Estimated number of detected pentaquarks per day.

\begin{tabular}{l|l|l}
\hline \hline & Untagged & Tagged \\
\hline$P_{c}(4380)$ & $24-750$ & $10-330$ \\
$P_{c}(4450)$ & $35-1100$ & $14-440$
\end{tabular}

\section{Conclusions}

Clarifying the nature of the recently discovered LHCb hidden-charm pentaquark peaks requires further experimental investigation. The energies and the luminosities accessible for the CLAS12+Forward Tagger detector permit detailed studies of these resonances, by measuring the photoproduction reaction $\gamma p \rightarrow P_{c} \rightarrow p J / \psi$. In CLAS12, this process is accessed trough low- $Q^{2}$ electron scattering on a fixed $\mathrm{LH}_{2}$ target, using both the "untagged" and the "tagged" techniques. The complementarity of these approaches, the first being characterized by a higher effective luminosity, and the second by a better $p J / \psi$ invariant mass resolution, permits to efficiently measure $J / \psi$ photoproduction near threshold, identify possible pentaquark states if the branching fraction for the $P_{c} p J / \psi$ decay is of the order of $1 \%$ or larger.

\section{REFERENCES}

[1] U. Camerini et al., Phys. Rev. Lett. 35, 483 (1975).

[2] B. Gittelman et al., Phys. Rev. Lett. 35, 1616 (1975).

[3] R. Aaij et al. [LHCb Collaboration], Phys. Rev. Lett. 115, 072001 (2015).

[4] M. Battaglieri et al., Near Threshold $J / \psi$ Photoproduction and Study of LHCb Pentaquarks with CLAS12, 2017 (preprint).

[5] V. Kubarovsky, M.B. Voloshin, Phys. Rev. D 92, 031502(R) (2015).

[6] R. Chen et al., Phys. Rev. Lett. 115, 132002 (2015).

[7] L. Maiani et al., Phys. Lett. $B$ 749, 289 (2015).

[8] F.K. Guo et al., Phys. Rev. D 92, 071502(R) (2015).

[9] V.V. Anisovich et al., Pentaquarks and Resonances in the $p J / \psi$ Spectrum, 2015 (preprint).

[10] S.J. Brodsky et al., Phys. Lett. B 498, 23 (2001).

[11] K.A. Olive et al. [Particle Data Group], Chin. Phys. C 38, 090001 (2014).

[12] J. Sakurai, Phys. Rev. Lett. 22, 981 (1966). 